\title{
INFLUENCIA DE LA TOPOLOGÍA EN EL CONDICIONAMIENTO DE MATRICES DE REDES ELÉCTRICAS
}

\section{TOPOLOGIC INFLUENCE ON THE CONDITIONING OF MATRICES OF ELECTRIC NETWORKS}

\author{
Miguel Arias Albornoz $^{1} \quad$ Abnery Ortiz Riquelme $^{1} \quad$ Marcelo Aedo Ruz $^{1}$ \\ Recibido el 21 de marzo de 2006, aceptado el 26 de noviembre de 2007 \\ Received: March 21, $2006 \quad$ Accepted: November 26, 2007
}

\begin{abstract}
RESUMEN
Se estudia la influencia de la topología de redes eléctricas y alimentadores en el condicionamiento de las matrices asociadas a su modelado matemático. Se observa que el número de condición de estas matrices tiene una gran dependencia de la estructura de la red y que es afectado por el grado de enmallamiento de la red. Se incluyen resultados de simulaciones computacionales de distintos casos que permiten establecer una relación de consecuencia.
\end{abstract}

Palabras clave: Matrices de redes, condicionamiento numérico, estabilidad numérica.

\section{ABSTRACT}

The influence of the topology of electric networks and feeders on the condition number of the matrix associated to its mathematical model is studied. It is noted that the condition number of these matrices has a great dependence on the structure of the network and that it is affected by the network degree. Computer simulation results of different cases are included, which show the achievement of a relation of consequence.

Keywords: Network matrices, condition number, numerical stability.

\section{INTRODUCCIÓN}

El modelado de la red de un sistema eléctrico, operando en condiciones normales, se traduce generalmente en un conjunto de ecuaciones simultáneas con coeficientes constantes, que pueden ser representadas en forma matricial, dando lugar a las llamadas "matrices de redes". Un ejemplo de lo anterior es el conocido problema de flujo en sistemas eléctricos de potencia, donde se requiere resolver un conjunto de ecuaciones, empleando matrices tipo admitancia de barras. Para resolver el problema de flujo se requiere conocer las potencias netas inyectadas en las barras de la red (vector de datos), que son sensibles a la lectura de instrumentos. Cuando la matriz del modelo es mal condicionada, la imprecisión en la lectura de datos puede generar problemas de inestabilidad numérica en la solución del sistema de ecuaciones, entregando soluciones erróneas, tanto a los operadores como a los sistemas de control, lo que puede afectar la toma de decisiones.
En la escasa literatura relacionada con el tema, el problema de mal condicionamiento en las matrices de redes se aborda solo parcialmente; sin embargo, las publicaciones revisadas contienen interesantes aportes sobre el tema. En 1983, Gu, Clements y otros [1] presentan un análisis de las fuentes de mal condicionamiento en el problema de estimación de estado y proponen una alternativa al método de solución de Peters y Wilkinson, para solucionar el problema de mal condicionamiento sin pérdida de la dispersidad de la matriz. Además, proponen un mejoramiento en el condicionamiento de la matriz G (usada en estimación de estado). En una contribución posterior de Arias y Quintana [2] se muestra una interpretación de este método, que permite tratar el problema de estimación como un problema de flujo de potencia de una red equivalente. En [3] se introduce un algoritmo recursivo para resolver sistemas lineales altamente mal condicionados, con buena precisión del error. Un nuevo algoritmo para resolver sistemas de ecuaciones mal condicionadas es presentado en [4], donde se propone un

\footnotetext{
1 Departamento de Ingeniería Eléctrica. Universidad de Santiago de Chile. Avenida Ecuador 3519. Casilla 10233. Santiago, Chile. E-mail: m-arias@usach.cl
} 
método radicalmente distinto a los tradicionales; primero se mejora el condicionamiento del sistema de ecuaciones y luego se resuelve. La solución aproximada es modificada para obtener la solución exacta. En [5] se presenta un método algebraico que usa la factorización triangular de la matriz de ganancia simétrica para determinar las islas observables, a partir de un conjunto de mediciones para supervisión de un sistema de potencia. R. Ebrahimian y R. Baldick en [6] desarrollan fórmulas para el número de condición del problema de estimación de estado como una función con diferente tipo y número de medidas. En [7] se destaca la importancia del condicionamiento de matriz jacobiana en la validación de modelos de redes neuronales. En [8] se estudia el problema de ubicación de puntos de medición para fines de estimación de estado en sistemas de potencia, usando el condicionamiento de la matriz del modelo de medición como criterio para evaluar la calidad de la configuración resultante. Finalmente, en [9], se estudia el condicionamiento numérico de la matriz de mínimos cuadrados asociada a redes libremente escaladas, utilizando un límite teórico inferior para identificar casos mal condicionados.

La revisión de la bibliografía relacionada con el tema ha permitido concluir que la influencia de la topología de la red eléctrica sobre el condicionamiento de las matrices resultantes de su modelado y la estabilidad numérica de las soluciones, es un problema poco estudiado. Buscando obtener respuesta a esta situación se han realizado estudios con sistemas eléctricos de diferente topología inicial, modificando paso a paso la topología de la red, y evaluando su efecto sobre el número de condición de la matriz resultante. Con el objetivo de simplificar el análisis, pero sin pérdida de generalidad, se ha trabajado con la matriz B del problema de flujo DC. Para cada experimento se construyeron gráficos que muestran la tendencia del número de condición en función del grado de enmallamiento de la red.

\section{PLANTEAMIENTO DEL PROBLEMA}

El modelo básico del Flujo DC establece una relación lineal entre potencias y ángulos en los nudos de una red eléctrica, a través de la siguiente ecuación:

$$
P=B \theta
$$

Donde:

$\mathrm{P}$ : vector de potencias netas inyectadas en las barras del sistema (n-1 x 1)
B : matriz (n-1 x n-1), que tiene una estructura simétrica y dispersa, igual a la matriz admitancia de barras.

$\theta$ vector de ángulos de barras del sistema, sin considerar la referencia $(\mathrm{n}-1 \times 1)$.

\section{Condicionamiento numérico de una matriz}

El sistema de ecuaciones lineales (1) tiene solución única cuando det $(B) \neq 0$. Para estudiar la sensibilidad de la solución $\theta$, con respecto a pequeñas perturbaciones en el vector de datos $(P)$, se considera el siguiente sistema perturbado, denotando con $\Delta$ los incrementos:

$$
B(\theta+\Delta \theta)=P+\Delta P
$$

Restando (1), se obtiene:

$$
B \cdot \Delta \theta=\Delta P \quad \text { ó } \quad \Delta \theta=B^{-1} \cdot \Delta P
$$

El vector de error absoluto $\Delta \theta$ no es necesariamente pequeño en comparación al vector $\theta$. En efecto, tomando normas:

$$
\begin{aligned}
& \|\Delta P\|=\|B \cdot \Delta \theta\| \leq\|B\| \cdot\|\Delta \theta\|, \\
& \|\Delta \theta\|=\left\|B^{-1} \cdot \Delta P\right\| \leq\left\|B^{-1}\right\| \cdot\|\Delta P\|
\end{aligned}
$$

Se obtiene,

$$
\frac{\|\Delta P\|}{\|B\| \cdot\|\theta\|} \leq \frac{\|\Delta \theta\|}{\|\theta\|} \leq \frac{\left\|B^{-1}\right\| \cdot\|\Delta P\|}{\|\theta\|}
$$

Tomando ahora las normas del sistema de ecuaciones (1), se tiene,

$$
\|P\|=\|B \cdot \theta\| \leq\|B\| \cdot\|\theta\|,\|\theta\|=\left\|B^{-1} P\right\| \leq\left\|B^{-1}\right\| \cdot\|P\|
$$

Combinando las expresiones (5) y (6):

$$
\frac{1}{\|B\| \cdot\left\|B^{-1}\right\|} \frac{\|\Delta P\|}{P} \leq \frac{\|\Delta \theta\|}{\|\theta\|} \leq\|B\| \cdot\left\|B^{-1}\right\| \frac{\|\Delta P\|}{P}
$$

El número:

$$
\kappa(B)=\|B\| \cdot\left\|B^{-1}\right\|
$$


Se denomina número de condición.

En relación con el número de condición se puede observar lo siguiente:

1. El número de condición de B está acotado inferiormente por 1

$$
1=\|I\|=\left\|B \cdot B^{-1}\right\| \leq\|B\| \cdot\left\|B^{-1}\right\|=\kappa(B)
$$

2. El número de condición es un buen predictor del condicionamiento de un sistema de ecuaciones $\mathrm{Ax}=\mathrm{b}$, cuando los coeficientes de A no varían mucho en magnitud. Una matriz diagonal siempre será bien condicionada.

3. Si el número de condición es muy próximo a 1 , perturbaciones muy pequeñas en $b$ conducen $a$ pequeñas perturbaciones en $\mathrm{x}$. Por el contrario, si $\kappa(B)>>1$, entonces pequeñas perturbaciones en $\mathrm{b}$ pueden producir grandes variaciones en $\mathrm{x}$.

4. Como el número de condición depende de la norma matricial, es conveniente especificar ésta, para evitar confusiones.

\section{SIMULACIÓN}

Con el objetivo de estudiar el efecto del enmallamiento de la red sobre el condicionamiento de la matriz $\mathrm{B}$, se ha estudiado el comportamiento del número de condición en sistemas de prueba con redes eléctricas de topología radial, y también con redes enmalladas, aplicando norma euclidiana. A partir de los resultados obtenidos es posible visualizar un patrón de tendencia, el que se confirma con los gráficos representativos de cada caso.

\section{Caso 1: Efecto sobre Redes Radiales}

Agregando líneas y barras en forma secuencial se construye un sistema radial puro, hasta obtener un sistema de 20 barras y 19 líneas. Posteriormente, también en forma secuencial, se agregaron líneas aleatoriamente, con el objetivo de construir ahora una red parcialmente enmallada, manteniendo el número de barras, resultando las indicadas en la tabla 1. El proceso de construcción secuencial de la red y su posterior enmallamiento se ilustra en el diagrama unilineal de la figura 1, donde las líneas continuas representan la parte radial mientras que las líneas punteadas la transforman en red enmallada. En cada etapa de construcción de la red se determinó el número de condición de la matriz $\mathrm{B}$, obteniendo los resultados que se muestran en la figura 2 .
Tabla 1. Líneas agregadas en red radial de 20 barras.

\begin{tabular}{|c|c|c|c|c|c|}
\hline Línea & \multicolumn{2}{|c|}{ Entre barras } & Línea & \multicolumn{2}{c|}{ Entre barras } \\
\hline 21 & 7 & 16 & 26 & 5 & 2 \\
\hline 22 & 13 & 19 & 27 & 19 & 7 \\
\hline 23 & 4 & 2 & 28 & 9 & 2 \\
\hline 24 & 4 & 19 & 29 & 11 & 8 \\
\hline 25 & 12 & 6 & 30 & 5 & 20 \\
\hline
\end{tabular}

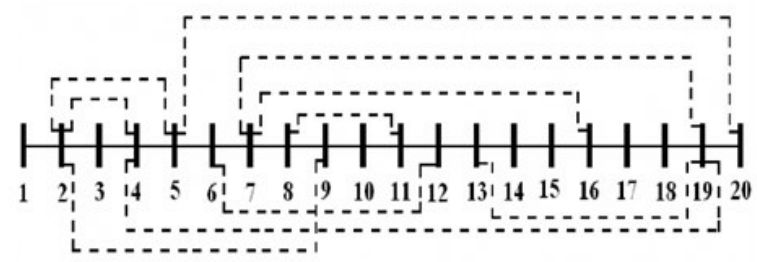

Figura 1. Diagrama unilineal red de 20 barras.

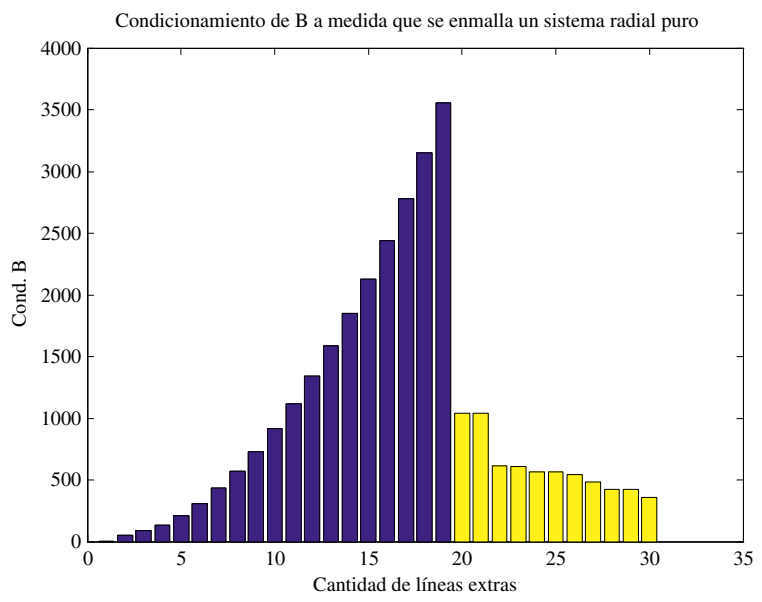

Figura 2. Efecto del enmallamiento en el condicionamiento de la matriz $\mathrm{B}$ de una red de 20 barras.

Para complementar este resultado se realizaron simulaciones con otros dos sistemas, con estructura radial de 50 y 70 barras. De manera análoga a lo realizado en el caso anterior, en forma secuencial se agregaron 20 ramas al sistema de 50 barras y 40 ramas al sistema de 70 barras, según se indica en tablas 2 y 3 . Los correspondientes diagramas unilineales pueden ser fácilmente reconstruidos a partir del procedimiento que se desprende del sistema de 20 barras.

En los gráficos de las figuras 2, 3 y 4 se muestran los resultados del proceso de enmallamiento de estas redes, donde las barras grises representan el condicionamiento del sistema radial puro y las barras de tono más claro el condicionamiento del sistema al introducir líneas extras para enmallar la red. 
Tabla 2. Líneas agregadas en red radial de 50 barras.

\begin{tabular}{|c|c|c|c|c|c|}
\hline Línea & \multicolumn{2}{|c|}{ Entre barras } & Línea & \multicolumn{2}{c|}{ Entre barras } \\
\hline 51 & 39 & 17 & 61 & 29 & 41 \\
\hline 52 & 45 & 18 & 62 & 50 & 42 \\
\hline 53 & 5 & 13 & 63 & 47 & 36 \\
\hline 54 & 11 & 15 & 64 & 9 & 22 \\
\hline 55 & 12 & 50 & 65 & 35 & 28 \\
\hline 56 & 8 & 50 & 66 & 34 & 28 \\
\hline 57 & 44 & 49 & 67 & 40 & 24 \\
\hline 58 & 25 & 28 & 68 & 11 & 7 \\
\hline 59 & 7 & 12 & 69 & 28 & 49 \\
\hline 60 & 20 & 2 & 70 & 49 & 29 \\
\hline
\end{tabular}

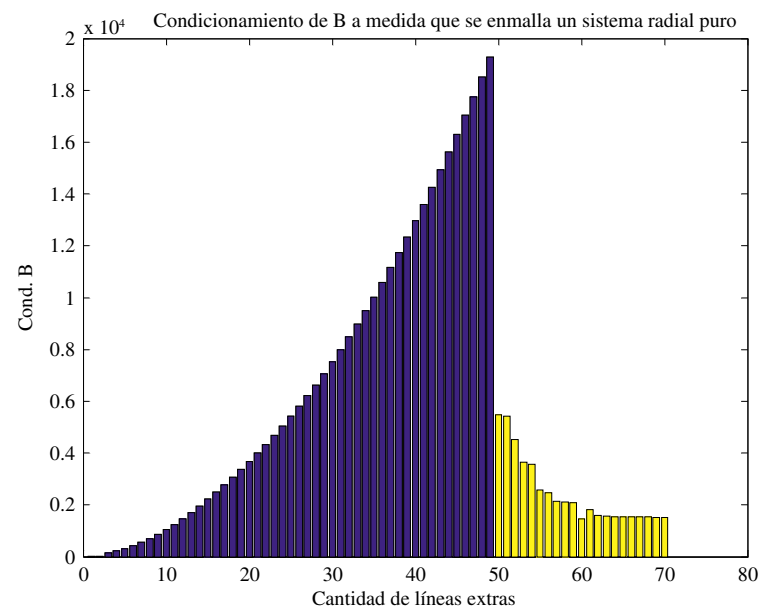

Figura 3. Efecto del enmallamiento en el condicionamiento de una red radial de 50 barras.

Tabla 3. Líneas agregadas en red radial de 70 barras.

\begin{tabular}{|c|c|c|c|c|c|}
\hline Línea & \multicolumn{2}{|c|}{ Entre barras } & Línea & \multicolumn{2}{c|}{ Entre barras } \\
\hline 71 & 10 & 14 & 91 & 48 & 61 \\
\hline 72 & 34 & 18 & 92 & 43 & 17 \\
\hline 73 & 49 & 3 & 93 & 48 & 27 \\
\hline 74 & 21 & 54 & 94 & 48 & 32 \\
\hline 75 & 55 & 23 & 95 & 12 & 5 \\
\hline 76 & 54 & 20 & 96 & 68 & 59 \\
\hline 77 & 63 & 5 & 97 & 8 & 68 \\
\hline 78 & 34 & 69 & 98 & 21 & 57 \\
\hline 79 & 33 & 28 & 99 & 70 & 41 \\
\hline 80 & 48 & 46 & 100 & 60 & 15 \\
\hline 81 & 49 & 59 & 101 & 69 & 23 \\
\hline 82 & 51 & 18 & 102 & 66 & 3 \\
\hline 83 & 18 & 41 & 103 & 30 & 58 \\
\hline 84 & 56 & 58 & 104 & 39 & 42 \\
\hline 85 & 23 & 47 & 105 & 34 & 23 \\
\hline 86 & 23 & 65 & 106 & 43 & 18 \\
\hline 87 & 26 & 66 & 107 & 18 & 42 \\
\hline 88 & 11 & 37 & 108 & 1 & 51 \\
\hline 89 & 19 & 50 & 109 & 57 & 67 \\
\hline 90 & 42 & 25 & 110 & 38 & 53 \\
\hline
\end{tabular}

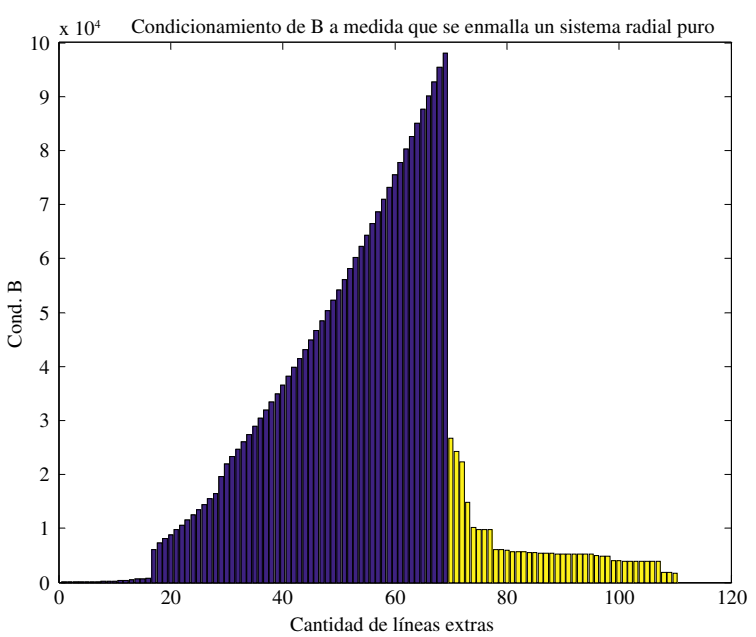

Figura 4. Efecto del enmallamiento en el condicionamiento de una red radial de 70 barras.

En los tres sistemas radiales estudiados se observa que el condicionamiento de la matriz $\mathrm{B}$ se incrementa exponencialmente con el número de barras cuando se mantiene el sistema radial puro; sin embargo, al producir enmallamiento sobre estos sistemas se verifica una gran reducción del número de condición, fenómeno que resulta independiente del número de barras. También se aprecia que el condicionamiento disminuye rápidamente al inicio del enmallamiento, y que después el número de condición se mantiene prácticamente constante, independiente del número de líneas extras.

\section{Caso 2: Efecto sobre Redes Enmalladas}

En este caso se experimentó, en primer lugar, con la red eléctrica del sistema IEEE de 14 barras. El proceso de enmallamiento de esta red se ha realizado adicionando líneas de manera aleatoria para aumentar el grado de enmallamiento, según se indica en la tabla 4. En figura 5, la red original se destaca con trazo continuo y con trazo segmentado se indican las líneas extras.

Tabla 4. Líneas agregadas en red IEEE de 14 barras.

\begin{tabular}{|c|c|c|c|c|c|}
\hline Línea & \multicolumn{2}{|c|}{ Entre barras } & Línea & \multicolumn{2}{c|}{ Entre barras } \\
\hline 1 & 5 & 9 & 6 & 13 & 9 \\
\hline 2 & 14 & 10 & 7 & 6 & 2 \\
\hline 3 & 7 & 1 & 8 & 6 & 10 \\
\hline 4 & 8 & 13 & 9 & 12 & 5 \\
\hline 5 & 3 & 11 & 10 & 14 & 1 \\
\hline
\end{tabular}




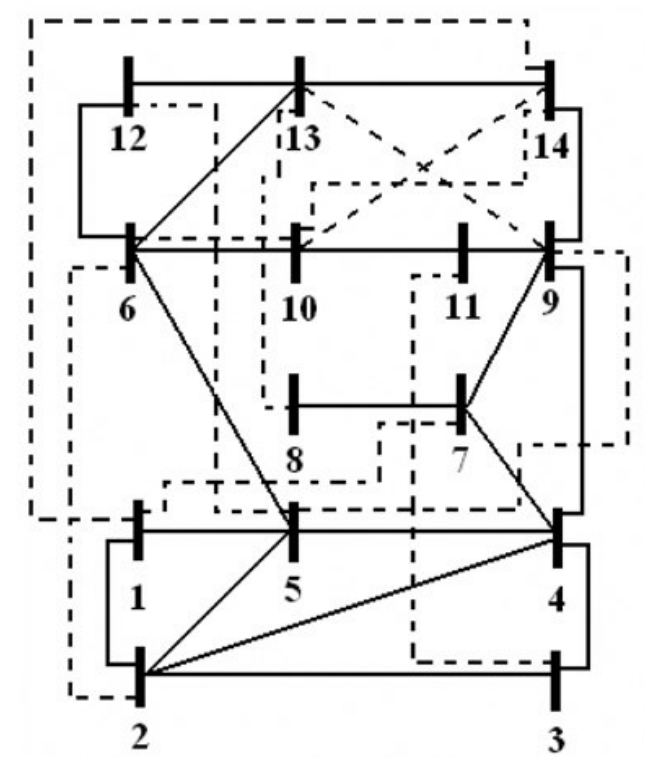

Figura 5. Diagrama unilineal sistema IEEE - 14 barras.

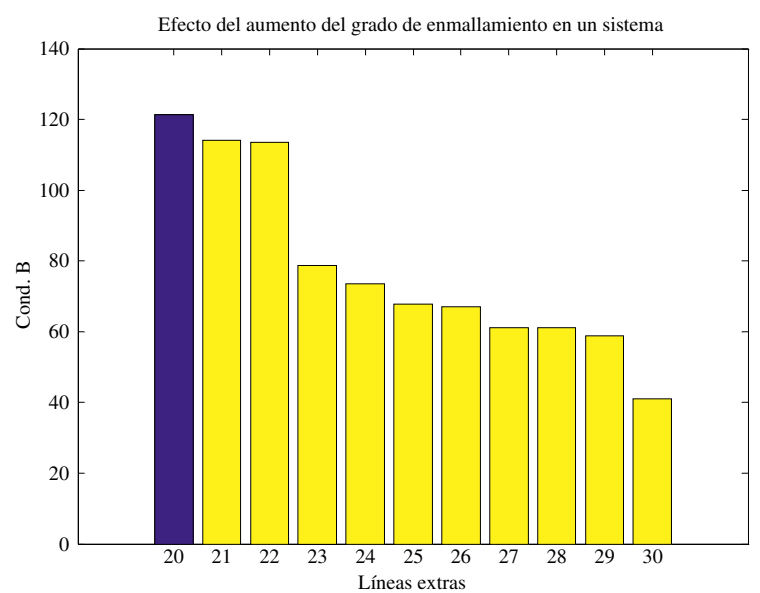

Figura 6. Efecto del enmallamiento en el condicionamiento de la red de 14 barras.

Como se puede observar en figura 6 , el condicionamiento de la matriz B tiende a disminuir a medida que aumenta el enmallamiento de la red, de modo similar a lo que ocurre en el Caso 1; por lo tanto, es de esperar que el condicionamiento sea más bajo cuando el sistema está completamente enmallado. Sin embargo, en este caso el número de condición muestra una tendencia a saturación, es decir, el condicionamiento de la matriz B llega a ser prácticamente independiente del número de líneas que se agregan.
Para complementar, se muestran resultados obtenidos con los sistemas IEEE enmallados de 30 y de 57 barras, en los cuales se agregaron líneas en forma secuencial, generadas aleatoriamente, de acuerdo a lo indicado en las tablas $5 \mathrm{y}$ 6 , repectivamente. En cada caso se determinó el número de condición de la matriz $\mathrm{B}$, graficando los resultados en función del número de líneas extras, como se muestra en las figuras 7 y 8 , respectivamente. En las figuras 6, 7 y 8, la barra en gris representa el condicionamiento de la matriz B correspondiente al sistema original, y las barras de tonos claros muestran el condicionamiento de la matriz B cuando se aumenta el grado de enmallamiento de la red.

Tabla 5. Líneas agregadas en red enmallada de 30 barras.

\begin{tabular}{|c|c|c|c|c|c|}
\hline Línea & \multicolumn{2}{|c|}{ Entre barras } & Línea & \multicolumn{2}{c|}{ Entre barras } \\
\hline 1 & 9 & 1 & 11 & 24 & 6 \\
\hline 2 & 2 & 29 & 12 & 30 & 5 \\
\hline 3 & 29 & 24 & 13 & 2 & 19 \\
\hline 4 & 16 & 5 & 14 & 17 & 19 \\
\hline 5 & 23 & 18 & 15 & 13 & 23 \\
\hline 6 & 25 & 30 & 16 & 3 & 19 \\
\hline 7 & 8 & 11 & 17 & 11 & 20 \\
\hline 8 & 16 & 15 & 18 & 25 & 6 \\
\hline 9 & 1 & 22 & 19 & 13 & 5 \\
\hline 10 & 21 & 12 & 20 & 16 & 25 \\
\hline
\end{tabular}

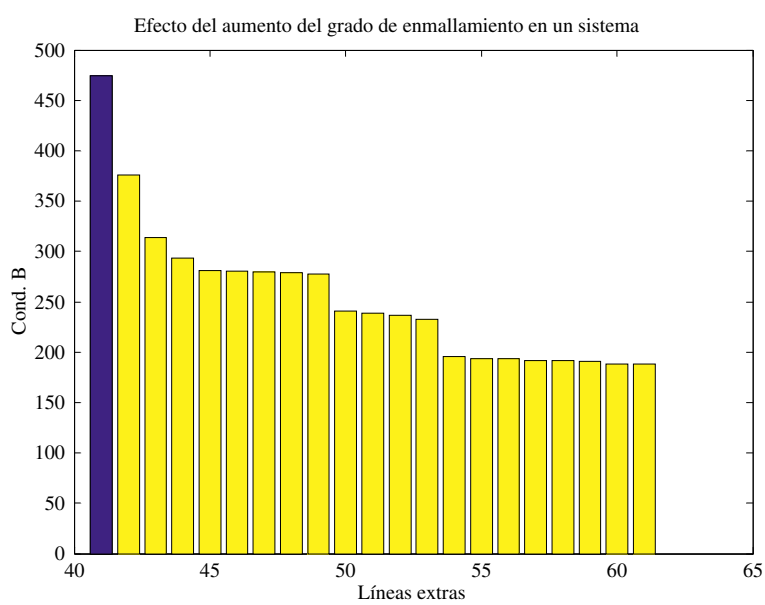

Figura 7. Efecto del enmallamiento en el condicionamiento de la red eléctrica de 30 barras. 
Tabla 6. Líneas agregadas en red enmallada de 57 barras.

\begin{tabular}{|c|c|c|c|c|c|}
\hline Línea & \multicolumn{2}{|c|}{ Entre barras } & Línea & \multicolumn{2}{c|}{ Entre barras } \\
\hline 1 & 24 & 52 & 16 & 8 & 3 \\
\hline 2 & 26 & 10 & 17 & 23 & 55 \\
\hline 3 & 40 & 8 & 18 & 3 & 38 \\
\hline 4 & 31 & 39 & 19 & 13 & 36 \\
\hline 5 & 5 & 34 & 20 & 14 & 17 \\
\hline 6 & 50 & 10 & 21 & 46 & 55 \\
\hline 7 & 14 & 22 & 22 & 16 & 10 \\
\hline 8 & 34 & 26 & 23 & 57 & 43 \\
\hline 9 & 57 & 9 & 24 & 12 & 57 \\
\hline 10 & 50 & 22 & 25 & 16 & 41 \\
\hline 11 & 46 & 57 & 26 & 50 & 25 \\
\hline 12 & 6 & 50 & 27 & 14 & 32 \\
\hline 13 & 5 & 19 & 28 & 53 & 55 \\
\hline 14 & 30 & 57 & 29 & 4 & 7 \\
\hline 15 & 44 & 7 & 30 & 4 & 31 \\
\hline
\end{tabular}

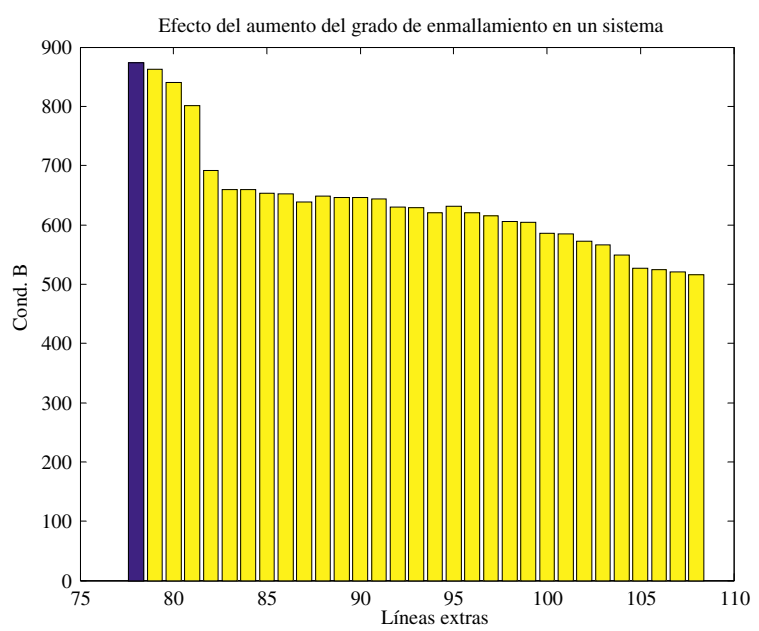

Figura 8. Efecto del enmallamiento en el condicionamiento de la red eléctrica de 57 barras.

\section{CONCLUSIONES}

En este trabajo se han realizado experimentos de simulación para determinar el efecto de la topología de una red eléctrica sobre el número de condición de la matriz $\mathrm{B}$ del modelo de flujo de potencia DC. Se ha trabajado con redes de topología radial y con redes enmalladas. En ambos casos se han agregado líneas adicionales para producir enmallamiento en el caso de las redes radiales, y para incrementar el grado de enmallamiento en el caso de redes enmalladas.
Con respecto a los sistemas de topología radial pura, los resultados obtenidos muestran que el número de condición de la matriz $\mathrm{B}$ se incrementa rápidamente a medida que aumenta el número de barras. También se aprecia un gran impacto en la reducción del número de condición cuando se produce enmallamiento del sistema radial, aunque sea solamente a través de una línea. Sin embargo, el gran efecto inicial en el condicionamiento de la matriz $\mathrm{B}$ disminuye rápidamente con el número de líneas adicionadas en la red, hasta un punto denominado zona de saturación.

Un fenómeno muy similar ocurre cuando el experimento se realiza a partir de una red de topología ya enmallada. En efecto, los resultados obtenidos permiten verificar que el condicionamiento de la matriz B mejora cuando aumenta el grado de enmallamiento de la red, pero su efecto no resulta comparable al del caso radial. También se observa una zona de saturación, a partir de la cual el número de condición de $\mathrm{B}$ se mantiene prácticamente constante con el incremento de líneas a la red.

En ambos casos se aprecia que el número de condición de la matriz $\mathrm{B}$ tiende a un mismo nivel al final del proceso. La diferencia entre ambos casos radica en que el condicionamiento de un sistema radial crece a medida que la red aumenta de tamaño, mientras mantenga una topología radial pura. Al producir enmallamiento en una red radial pura, se produce una considerable reducción del número de condición de la matriz B. Por otra parte, al incrementar el grado de enmallamiento de una red ya enmallada, sólo se observa una disminución leve del número de condición.

Se considera que es importante tener en cuenta estos resultados para evitar errores en las decisiones, introducidos por problemas de condicionamiento de matrices no detectados por los algoritmos de solución. Por lo anterior es conveniente realizar análisis de sensibilidad, para identificar errores graves que pueden ser provocados por un mal condicionamiento de las matrices del modelo.

\section{REFERENCIAS}

[1] J.W. Gu, K.A. Clements, G.R. Krumpholz and P.W. Davis. "The solution of ill-conditioned power system state estimation problems via the method of Peters and Wilkinson". IEEE Transaction on PAS. Vol. PAS-102 No 10, pp. 3473-3480. October 1983. 
[2] M. Arias and V. Quintana. "Equivalent network approach for the analysis of the gain matrix". Proceedings North American Power Symposium, pp. 371-375. Manhattan-Kansas. USA. September 1994.

[3] J. Wang, X. Yu, N. K. Loh, Z. Qin and W. C. Miller. "Solving linear algebraic equations without error". IEEE Signal. Processing Letters. Vol. 1 NN$^{\circ}$ 3. March 1994.

[4] H. Joong, K. Choi, H.B. Lee and Y. Hahn. "A new algorithm for solving ill-conditioned linear systems". IEEE Transactions on Magnetics. Vol. 32 No 3. May 1996.

[5] B. Gou and A. Abur. "A direct numerical method for observability analysis". IEEE Transactions on Power Systems. Vol. $15 \mathrm{~N}^{\circ}$ 2, pp. 625-630. May 2000.
[6] R. Ebrahimian and R. Baldick. "State estimator condition number analysis". IEEE Transaction on Power Systems. Vol. 16 N $^{\circ}$ 2, pp. 273-279. May 2001.

[7] I. Rivals and L. Personnaz. "Jacobian conditioning analysis for model validation". Neural Computation. Vol. $16 \mathrm{~N}^{\circ} 2$, pp. 401-418. February 2004.

[8] C. Rakpenthai, S. Premrudeepreechacham, S. Uatrongjit and N.R. Watson. "Measurement placement for power system state estimation using decomposition technique". Electric Power Systems Research Vol. 75 No 1, pp. 41-49. July 2005.

[9] G. Acosta, M. Grana and J.P. Pinasco. "Condition number and scale free graphs". European Phisical Journal B. Vol. $53 \mathrm{~N}^{\circ} 3$, pp. 381-385. October 2006. 